View at Publisher

Quarterly Review of Economics and FinanceVolume 69, August 2018, Pages 155-173

\title{
An empirical application of a stochastic volatility model with GH skew Student's t-distribution to the volatility of Latin-American stock returns(Article)
}

- Lengua Lafosse, P.a, Email Author,

- Rodríguez, G. ${ }^{\circ}$ Email Author

- View Correspondence (jump link)

- aUniversidad Peruana de Ciencias Aplicadas - UPC, Peru

- $\quad$ 'Pontificia Universidad Católica del Perú, Peru

- Faculty of Economics, Universidad Peruana de Ciencias AplicadasUPC, Prolongación Primavera 2390, Monterrico, Lima, 33, Peru

\begin{abstract}
View references (62)
Using daily stocks returns data of a set of Latin-American countries (Argentina, Brazil, Chile, Mexico and Peru) for the sample period 1996:01-2013:12, we estimate a stochastic volatility model incorporating both leverage effects and skewed heavy-tailed disturbances through of the GH Skew Student's tdistribution based on Bayesian estimation method proposed by Nakajima and Omori (2012). Two alternative models are estimated, one using an alternative Skew Student's t-distribution and the other using a symmetric Student's tdistribution. The results suggest the presence of leverage effects in all markets except for Peru where the evidence is unclear. In addition, there is evidence of asymmetries and heavy tails in the Argentina and S\&P500 markets while in the other countries there is no robust evidence of such characteristics. Using the Bayes factor, the results indicate that the SVGHSkewt model dominates the other two models for the cases of Peru, Argentina, Brazil and S\&P500 whereas the simple SVt model is preferred for the markets of Mexico and Chile. Similar findings are obtained after performing a robustness analysis regarding the priors of the parameters associated with the skewness and the tails of the distribution. (C) 2018 Board of Trustees of the University of Illinois
\end{abstract}

SciVal Topic Prominence

Topic: Stochastic volatility model | Stochastic volatility | MCMC Prominence percentile: 74.029

- ISSN: 10629769

- Source Type: Journal

- Original language: English 
- DOI: 10.1016/j.qref.2018.01.002

- Document Type: Article

- Publisher: Elsevier B.V. 